\title{
« And sent him homeward tae think again »: UCS, Heath et la résurgence du militantisme écossais
}

"And sent him homeward tae think again": Upper Clyde Shipbuilders, Edward Heath, and the Resurgence of Scottish Syndicalism

\section{Laëtitia Langlois}

\section{(2) OpenEdition}

\section{Journals}

Édition électronique

URL : https://journals.openedition.org/etudesecossaises/338

DOI : 10.4000/etudesecossaises.338

ISSN : 1969-6337

Éditeur

UGA Éditions/Université Grenoble Alpes

Édition imprimée

Date de publication : 31 mars 2011

Pagination : 93-113

ISBN : 978-2-84310-191-5

ISSN : $1240-1439$

\section{Référence électronique}

Laëtitia Langlois, « «And sent him homeward tae think again » : UCS, Heath et la résurgence du

militantisme écossais », Études écossaises [En ligne], 14 | 2011, mis en ligne le 31 mars 2012, consulté le 14 avril 2023. URL : http://journals.openedition.org/etudesecossaises/338 ; DOI : https://doi.org/ 10.4000/etudesecossaises.338 


\section{«And sent him homeward tae think again»: Upper Clyde Shipbuilders, Edward Heath et la résurgence du militantisme écossais}

UCS, trois lettres qui renvoient à une entreprise mythique, Upper Clyde Shipbuilders, et à un célèbre slogan, «Unity Creates Strength». Upper Clyde Shipbuilders est indissociablement lié au mouvement ouvrier qui vit le jour en juin 1971 après l'annonce faite par John Davies, alors ministre de l'Industrie du gouvernement conservateur, de fermer UCS.

Les conservateurs voulaient faire d'UCS le symbole de leur nouvelle politique industrielle anti-«canards boiteux ${ }^{1} »$ mais c'est UCS qui changera le cours de leur politique et le cours de l'histoire de ce gouvernement. UCS représente le point de départ des retours en arrière de Heath et de son retour à une politique modérée, interventionniste et collectiviste. Comment un gouvernement doté d'un mandat électoral clair pour mettre fin à certaines pratiques dispendieuses a-t-il pu autant infléchir sa politique industrielle après l'épisode UCS? À la brutalité et l'indifférence du gouvernement conservateur, les ouvriers des chantiers navals de Clydeside ont répondu par la mobilisation, la solidarité et le dévouement. L'épisode UCS a vu la résurgence d'un militantisme écossais radical et a vu l'Écosse renouer avec ses traditions de mobilisation massive de la classe ouvrière héritées du temps de Red Clydeside. Comment s'est opérée cette résurgence? Comment s'est-elle manifestée? Nous verrons qu'avec UCS nous assistons à une résurgence pluridimensionnelle. UCS invoque de nombreux points de résurgence dans la mesure où ce mouvement dépasse de loin le cadre de la mobilisation ouvrière. UCS a fait resurgir un passé, une histoire, des traditions, des valeurs, des convictions qui ont réveillé en Écosse un souffle et une force qui sont allés bien au-delà du mouvement ouvrier. Nous verrons qu'avec la résurgence du militantisme écossais, c'est toute une nation écossaise qui s'est réveillée et qui a pris conscience de sa force.

1. John Davies utilisa cette formule la première fois aux Communes le 4 Novembre 1970. 


\section{Entre résurgence et décadence, le sort d'UCS et de l'Écosse dans les années 1960-1970}

Thomas Devine décrit la construction navale dans la région de la Glyde comme «un emblème de l'Écosse et un symbole fort du passé glorieux de l'industrie écossaise ${ }^{2} »$ (Devine, The Scottish Nation, p. 584) Au début du $\mathrm{Xx}^{\mathrm{e}}$ siècle, l'Écosse faisait partie des leaders mondiaux dans la construction de bateaux et jouissait d'une réputation internationale de prestige et de qualité qui faisait la fierté des Écossais et des ouvriers des chantiers navals. Le secteur avait cependant été durement frappé par la récession de l'entre-deux-guerres et avait connu une sérieuse baisse d'activité et un fort taux de licenciement à la suite des fermetures d'usines qui avaient plongé la région dans le marasme économique, l'agitation sociale et la détresse humaine. La Seconde Guerre mondiale ainsi que la guerre de Corée avaient permis aux chantiers navals de la Clyde de connaître un court regain d'activité dans les années 1940 et 1950. La politique de réarmement offrait à l'industrie navale une nouvelle période de prospérité et de plein emploi. Ainsi dans les années 1950, 12\% des bateaux produits dans le monde étaient construits en Écosse et ce secteur employait, toujours dans les années 1950, plus de 27000 hommes, c'est-à-dire, le même taux qu'en 1900 (Knox, Industrial Nation, p. 254). Mais la lune de miel fut de courte durée et très vite l'Écosse fut rattrapée par la réalité économique et à partir des années 1960 William Knox note que «les phénomènes économiques visibles dans l'entre-deux-guerres refirent surface avec une violence accrue dans les années qui suivirent la fin de la guerre ${ }^{3}$ » (ibid.). Les années 1960 virent la résurgence des problèmes endémiques à l'économie écossaise et la crise toucha plus durement l'Écosse que le reste du Royaume-Uni. L'inflation galopante qui sévissait en GrandeBretagne était un des facteurs majeurs du ralentissement de l'économie. Les bateaux écossais coûtaient cher et n'étaient pas compétitifs face à des pays comme l'Allemagne, le Japon ou la Suède qui construisaient vite et meilleur marché (voir McGill, Crisis on the Clyde, p. 23-24). L'Écosse était incapable de résister face à cette concurrence étrangère, d'autant plus qu'au même moment le marché de la construction navale se réorganisait et se concentrait sur la construction de pétroliers qui exigeaient une infrastructure bien différente de celle requise pour la construction de paquebots ou de bateaux dont l'Écosse était spécialiste. Knox fait remarquer que l'Écosse perdit le leadership dans le marché faute d'avoir su

2. «a Scottish icon, a great symbol of the country's glorious industrial past».

3. «the economic trends observable in the inter-war period reasserted themselves with even greater ferocity in the decades after 1945 ». 
s'adapter aux nouvelles méthodes de construction et d'avoir modernisé ses chantiers. Résultat de cette résistance à la nouveauté et à la modernité : entre 1950 et 1968, le nombre de chantiers sur la Clyde passa de vingt-huit à sept et la main d'œuvre employée dans les chantiers navals baissa de moitié entre 1950 et 1978 (Buchan, The Right to Work, p. 25).

À la fin des années 1960, la construction navale en Écosse était une industrie en pleine déliquescence qui semblait complètement dépassée par la nouvelle ère industrielle qui se profilait à l'aube des années 1970. Les années 1970 marquèrent en effet l'entrée dans une nouvelle ère industrielle, l'ère de l'inbuilt obsolescence, guidée par de nouveaux modèles et de nouvelles exigences : la rapidité et la rentabilité primaient sur la qualité et le savoir-faire. Or le label Clyde-built était un label de prestige, un signe de qualité et de durabilité dont les ouvriers étaient fiers. Leurs années d'apprentissage avaient servi à leur enseigner une technique mais avaient aussi forgé un esprit qui était celui du travail bien fait et c'est à la lumière de cet élément qu'il est possible de jeter un regard différent sur l'attitude des ouvriers. Il ne s'agissait pas en effet d'un refus obstiné de se moderniser mais plutôt d'une résistance idéologique à une certaine vision de l'industrie et du travail industriel. Ces ouvriers ont refusé de se conformer aux diktats d'une nouvelle ère industrielle qui ne jurait que par la rapidité, le profit et le rendement, et c'est tout à leur honneur d'avoir voulu sauver leur savoir-faire et leurs traditions, mais leur entêtement leur a coûté cher. À la fin des années 1960, la construction navale subit une crise telle qu'Anthony Benn, le ministre de l'Industrie nomma un comité pour réfléchir à l'avenir de cette industrie. Le rapport Geddes, publié en 1966, énonçait deux recommandations principales pour garantir la survie de la construction navale en Écosse : tout d'abord, le regroupement des différentes entreprises en un seul et même groupe et ensuite le soutien financier de l'État aux chantiers navals.

Ainsi, sous l'impulsion de Tony Benn et sur les recommandations du rapport Geddes, naissait en février 1968 UCS, un consortium qui regroupait cinq entreprises : Yarrow, Stephens, Fairfields, Connels, John Browns. L'État était actionnaire à hauteur de 48,4\% et avait accordé un crédit sans intérêt de 5,5 millions de livres sur trois ans. Jack McGill juge cette somme dérisoire au regard des dettes accumulées par la compagnie (McGill, p. 21). Le directeur de l'époque, Anthony Hepper, avait dès le départ confié au gouvernement que la création d'UCS était une démarche sociale et en aucun cas financière, le but étant de maintenir un secteur qui employait un nombre considérable d'hommes et faisait vivre toute une région (ibid., p. 21). Anthony Hepper avait vu juste. L'entreprise, dans les mois qui suivirent sa création, ne montra aucun signe d'amélioration et les sommes demandées au gouvernement ne firent que 
s'accroître : 6 millions de livres en février 1968, 9 millions en mars 1969, et la somme record de 12 millions en mai 1969 (McGill, p. 21 et 30-38). Tony Benn déclara quelques années plus tard à ce sujet :

On en arriva au point où tous les vendredis il fallait se rendre en personne au ministère des Finances pour passer des coups de fil, et organiser des réunions pour débloquer les fonds. Sitôt avait-on donné de l'argent qu'il fallait en donner encore plus ${ }^{4}$. (Ibid., p. 40)

Benn décrit une situation ubuesque où UCS pouvait se permettre d'accepter des commandes peu rentables sachant pertinemment que le gouvernement lui porterait secours en cas de perte. Pourquoi ces aides massives? Pourquoi cette volonté gouvernementale de venir en aide à l'entreprise en dépit des sommes astronomiques que l'État devait verser pour garantir sa survie? Il faut considérer l'aide de l'État à UCS dans le contexte plus général du déclin de l'économie écossaise dans son ensemble. L'Écosse était une région sinistrée par le chômage. Entre 1960 et 1975 c'est dix mille hommes par an qui perdaient leur emploi dans cette seule région et le taux de chômage en Écosse était deux fois supérieur au reste du Royaume-Uni (Knox, p. 254). On comprend mieux l'aide massive de l'État dans ce contexte de crise économique et de chômage endémique. Les travaillistes étaient conscients du drame humain qui se jouait derrière le sort d'UCS : la fermeture de l'usine entraînerait dans son sillon la disparition de milliers d'emplois et la mort de toute une vallée. Comme l'avait fait remarquer The Glasgow Herald, «le coût économique et social — peu importe le coût politique — de la faillite d'UCS serait bien plus élevé que six millions de livres ${ }^{5}$ » (The Glasgow Herald, 12 juin 1971). Qu'allait devenir cette volonté politique avec le changement de gouvernement?

\section{Résurgence du laissez-faire}

En juin 1970, Edward Heath remplaçait Harold Wilson à Downing Street. Le gouvernement conservateur n'était pas dans une optique sociale. Les conservateurs avaient l'intention de mettre en place une nouvelle politique économique et industrielle articulée autour de deux points principaux : un programme de dénationalisation et la fin des subventions aux entreprises déficitaires. John Davies qui avait succédé à Tony Benn au

4. «It got to the point where one had to go every Friday to the Treasury almost personally, make telephone calls, and have meetings to clear the money. It was running out at the same time as it was going in.»

5. «The economic and social cost — never mind the political cost - of allowing UCS to go to the wall would be vastly greater than $£ 6$ million.» 
ministère de l'Industrie et de la Technologie donnait le ton de la nouvelle politique gouvernementale :

Nous pensons que le pays a un besoin vital que sa politique soit destinée à la grande majorité des gens qui ne sont pas des canards boiteux, qui n'ont pas besoin d'aide, qui sont capables de gérer seuls leurs propres intérêts [...]. La décadence nationale vient de cette habitude d'avoir traité les gens de ce pays comme s'ils étaient tous des canards boiteux ${ }^{6}$. (House of Commons Debate, Hansard, 4 novembre 1970, vol. 805/col. 1211-1212)

L'objectif de cette politique radicale était clair : accroître la compétitivité et encourager la modernisation des entreprises britanniques afin de préparer l'entrée dans la Communauté européenne. La croissance britannique dépendait avant tout de la bonne santé des entreprises et de leur capacité à s'ajuster aux nouvelles exigences pour relever le défi européen. Le gouvernement de Heath semblait convaincu des bienfaits d'une politique industrielle dans laquelle les entreprises les plus faibles étaient éliminées naturellement par la concurrence et les lois du marché. Une note de Davies sur l'avenir de la construction navale résume bien cette nouvelle philosophie :

Il n’y aura pas de subventions ou de soutiens accordés aux canards boiteux au détriment d'autres secteurs de l'industrie. Le Gouvernement de Sa Majesté veut des garanties en échange de son argent ${ }^{7}$. (National Archives, PREM 15/700)

Le gouvernement de Heath voulait se débarrasser de qu'il appelait les «canards boiteux» qui gangrénaient l'industrie britannique et pesaient sur les dépenses publiques. Mais est-ce que UCS était un «canard boiteux »? Au regard des chiffres, l'entreprise rentrait incontestablement dans cette catégorie. Son incapacité à atteindre l'équilibre budgétaire et à faire des profits malgré l'argent de l'État et un bon carnet de commandes, l'absentéisme chronique, et les retards de livraisons ${ }^{8}$, tous ces éléments réunis faisaient d'UCS une entreprise bien mal en point.

6. «We believe that the essential need of the country is to gear its policies to the great majority of people who are not lame ducks, who do not need a hand, who are quite capable of looking after their own interests $[\ldots]$. National decadence is the consequence of treating the whole country as though we were lame ducks. »

7. «There will be no element of subsidy or propping up lame ducks at the expense of other sectors of the industry. Her Majesty's Government will be obtaining value for their money.»

8. Le Queen Elizabeth II fut livré avec 105 jours de retard et fut surnommé «Le bateau de la honte» (voir McGill, p. 135). 
C'est ce qu'avait pointé du doigt Nicholas Ridley dans son rapport sur UCS rédigé en $1969^{9}$. Le Parti conservateur l'avait chargé de faire un état des lieux de l'entreprise : son rapport était accablant et mettait en lumière les faiblesses structurelles de l'entreprise. Ridley qualifiait UCS de «cancer rongeant toute la vie industrielle de Clydeside ${ }^{10}$ » et UCS était à ses yeux «un exemple parfait de l'absurdité de l'interventionnisme d'État $^{11}$ ». Ainsi, il préconisait purement et simplement l'arrêt des subventions pour laisser UCS aller à la faillite et conseillait ensuite de nommer «un boucher pour découper UCS et vendre (à bas prix) à Lower Clyde et à d'autres le capital d'UCS pour minimiser les risques de crise et d'agitation $^{12}{ }^{\prime}$ (The Guardian, 6 mai 1970). Le rapport Ridley n'a jamais servi de politique officielle aux conservateurs, mais dans la mesure où il fut nommé sous-secrétaire d'État à l'Industrie en 1970, on peut raisonnablement penser que l'avenir d'UCS était déjà plus ou moins joué d'avance dans l'esprit des conservateurs. Entre le rapport de Nicholas Ridley et le discours de John Davies aux communes, le Parti conservateur montrait un nouveau visage, celui d'un conservatisme radical et agressif qui ne jurait que par les mots compétitivité, efficacité, et modernisation, bien loin du torysme bienveillant et paternaliste des conservateurs de l'aprèsguerre.

Ainsi, quand le 9 juin 1971, Anthony Hepper annonça à Davies que l'entreprise devait faire face à des dettes qui s'élevaient à plus de 32 millions de livres à rembourser auprès de plus de mille créanciers à travers le monde, qu'elle était incapable de payer ses ouvriers et que de surcroît elle devait faire face à un déficit de 6 millions de livres, le sort d'UCS était connu d'avance (McGill, p. 84 et 88-89). Le 15 juin 1971, le couperet tomba. Davies annonça à la Chambre des communes qu'il n'aiderait pas l'entreprise et qu'il était temps de mettre fin à ce «bourbier d'incompétence subventionnée» qu'était $\mathrm{UCS}^{13}$. Il ajoutait :

La position du Gouvernement est que l'entreprise dans sa forme actuelle, criblée comme elle est de dettes, rongée par les déficits depuis sa création, ayant englouti et perdu quelque vingt millions de livres prêtées et accordées après arrangements avec le Gouvernement précédent, n’est pas en mesure de parvenir à un niveau de stabilité et de prospérité sans avoir recours à

9. Nicholas Ridley, «UCS Ltd, Confidential» (1969) : ce rapport fut publié dans The Guardian le 6 mai 1970.

10. «a cancer eating the whole of Clydeside industrial life».

11. «a prime example of the folly of Government Intervention».

12. "a Government "Butcher" to cut up UCS and to sell (cheaply) to Lower Clyde, and others, the assets of UCS, to minimise upheaval and dislocation».

13. «a morass of subsidised incompetence». 
l'aide gouvernementale. J'ai dû en arriver à la conclusion difficile que cette entreprise était peu susceptible dans un avenir proche d'avoir des chances de succès qui justifieraient que le Gouvernement verse une fois de plus d'importantes sommes d'argent pour la maintenir en vie ${ }^{14}$. (House of Commons Debate, Hansard, 14 juin 1971, vol. 819/col. 233-234)

Les conservateurs étaient cohérents avec leur politique et c'est une ligne de fermeté qui était affichée par le parti. Le 16 juin, Heath reçut les délégués syndicaux d'UCS à Downing Street et ne changea pas ses positions (National Archives, PREM 15/697). Par ailleurs, le rapport commandé par le gouvernement aboutissait aux mêmes conclusions et confortait Heath et Davies dans leurs décisions :

L'enseignement à tirer de cette affaire doit être que toute continuation d'Upper Clyde Shipbuilders dans sa forme actuelle serait totalement injustifiée et, de plus, pourrait causer des dégâts sérieux et considérables. Il est important que les leçons de cet échec soient claires et sans équivoque. Nous sommes conscients des conséquences sociales que le désastre d'UCS provoque. Mais il ne sert à rien de laisser croire qu'une autre tentative de reconstruction d'UCS ne serait autre qu'un expédient coûteux et temporaire de plus ${ }^{15}$. (Ibid., Report of the Advisory Group on Shipbuilding on the Upper Clyde)

Tous semblaient décidés à signer l'arrêt de mort d'UCS, mais c'était sans compter sur la détermination et l'opiniâtreté des 8500 ouvriers menacés de licenciement bien résolus à montrer aux hommes de Westminster que dans cette région et dans cette entreprise, comme le disait Jimmy Reid : «On ne construit pas seulement des bateaux, on construit des hommes intelligents et courageux et qui ne resteront pas les bras croisés ${ }^{16}$.» (McGill, p. 102)

14. «The Government's attitude is that the company in its present grouping, saddled as it is with debt, dogged by deficit since its inception, having absorbed and lost some $£ 20$ million lent and granted to it under arrangements by the former Government, is unlikely to achieve a state of stability and prosperity without having recourse to Government aid. I had to come to the painful conclusion that this concern was unlikely within the foreseeable future to reach any reasonable state of viability which would justify the Government in once more putting in a large sum of money to sustain it.»

15. "The conclusion must be that any continuation of Upper Clyde Shipbuilders in its present form would be wholly unjustified and, indeed, could cause serious and more widespread damage. It is important that the lessons of this failure are clear and unambiguous. We are aware of the social consequences that the UCS disaster causes. But there is no good pretending that another attempt at a reconstructed UCS would be anything but another costly and temporary makeshift. »

16. «We don't only build boats, we build men who have intelligence and guts and who will keep moving. » 


\section{La Résurgence d'une voix ouvrière}

\section{Résurgence d'un passé mythique}

Il a toujours existé une forte tradition de militantisme dans les chantiers navals de la Clyde, et depuis la Première Guerre mondiale ce secteur était connu pour la fréquence ainsi que l'ampleur souvent considérable de ses conflits sociaux. Quelles étaient les raisons de cet activisme syndical? C'est la question que pose William Knox dans Industrial Nation et pour lui les raisons sont multiples :

La réponse est à trouver dans une combinaison de facteurs locaux, ancrés dans les peurs, les valeurs et l'environnement des ouvriers, et de facteurs nationaux, liés aux politiques gouvernementales et à la restructuration du marché du travail. Les ouvriers qualifiés, notamment dans les grandes industries lourdes de l'ouest de l'Écosse, avaient développé un ensemble de mesures de protestations contre le patronat forgées par des décennies de chômage cyclique, de difficultés financières et de conflits sur le lieu de travail. Ces différents facteurs créèrent un climat de suspicion et d'antipathie mutuelle entre les deux camps du monde industriel ${ }^{17}$. (Knox, p. 288-289)

Knox décrit ici le sentiment partagé par beaucoup d'Écossais d'être en marge du débat et de la politique nationale. Ce sentiment de marginalisation a accru la puissance de la mobilisation et la volonté de se manifester en tant que groupe, car le rapport de force était au départ peu favorable aux ouvriers écossais. Ainsi, le militantisme écossais se distingue du militantisme anglais par son caractère plus radical et plus agressif, car il s'est nourri de la brutalité des licenciements dans les années 1920, notamment les licenciements massifs dans les chantiers navals de la Clyde auxquels nous faisions référence en première partie (Forster et Woolfson, The Politics of the UCS Work-In, p. 153-154). Les crises endémiques à l'économie écossaise ont instauré une tradition de militantisme qui s'est poursuivie bien au-delà des années 1920, comme en témoigne l'épisode d'UCS. Autre spécificité écossaise qui s'est ensuite étendue dans le reste du pays, cet activisme syndical a été porté par les délégués syndicaux (shop stewards) qui ont rapidement supplanté les leaders syndicaux et sont devenus le moteur central de la mobilisation ouvrière dans la région de la Clyde

17. «The answer lies in a combination of local factors, associated with worker fears, values and location, and national ones, associated with government economic policies and labour market restructuring. Skilled workers, particularly in the integrated heavy industries of the west of Scotland, had developed a set of industrial attitudes towards capital forged by decades of cyclical unemployment, economic hardship and workplace conflict. These experiences created a climate of suspicion and mutual loathing between the two sides of industry. » 
(ibid., p. 150). C'est eux qui ont fait du syndicalisme écossais un syndicalisme radical et cette tradition s'est perpétuée jusque dans les années 1970. Peut-on alors parler d'une résurgence du militantisme écossais s'il a toujours existé? Oui, mais peut-être serait-il plus approprié de parler de la résurgence d'une voix ouvrière comme au temps de Red Clydeside.

Red Clydeside fait référence aux deux décennies de mobilisation de la classe ouvrière et de conflits parfois violents avec les autorités britanniques qui ont secoué l'Écosse entre les deux guerres mondiales. Aujourd'hui, cette période a acquis une dimension légendaire, sans doute exagérée, mais qui témoigne néanmoins de la force de cet événement et de son impact sur l'imaginaire collectif. Les débats d'historiens ne manquent pas autour de ce mouvement et de la réalité de son ampleur, mais la réalité des historiens et la réalité populaire divergent fréquemment ${ }^{18}$. Red Clydeside échappe à l'historien dans la mesure où l'imaginaire collectif s'en est emparé, quelque soit l'ampleur véritable de cet épisode, il appartient désormais à la mémoire écossaise qui l'a érigé en mythe, en symbole de la résistance ouvrière. Tout combat a besoin de référents, de modèles sur lesquels s'appuyer, et cet épisode, incontestablement en est un. La puissance du mythe Red Clydeside est source d'inspiration pour des générations d'ouvriers qui se sentent appartenir à une lignée d'hommes qui disent non et qui résistent aux décisions des hommes de Londres, et il a sans doute contribué à générer un dynamisme militant au sein des hommes d'UCS qui pouvaient se reconnaître dans le combat de leurs aînés. Cependant, il est important de préciser que si les deux épisodes se font écho sur le plan symbolique, ils sont très différents sur le plan historique. Les méthodes employées et les revendications étaient bien différentes, mais la mobilisation était la même. Dans les deux cas, c'est toute une communauté qui était mobilisée pour défendre les intérêts de la classe ouvrière, comme l'ont montré les manifestations de soutien après l'annonce de John Davies de ne pas sauver UCS. Rarement les rues de Glasgow avaient connu telle mobilisation depuis l'entre-deux-guerres. Le 23 juin 1971, 150000 personnes arrêtèrent le travail et 50000 participèrent à la manifestation à Glasgow. Le 18 août, c'est 200000 personnes qui arrêtèrent le travail et 80000 qui participèrent à la manifestation qui ce jour là vit réunis aussi bien des membres du Parti communiste, du Parti travailliste, ou du Trades Union Congress (TUG) (Forster et Woolfson, p. 186 et 239). Mais au-delà de la mobilisation exceptionnelle, c'est la tactique employée pour résister au gouvernement qui a marqué

18. Pour une historiographie de Red Clydeside, voir notamment I. McLean, The Legend of Red Clydeside, Édimbourg, John Edward, 1983, et R. Duncan et A. McIvor (eds), Militant Workers: Labour and Class Conflict on the Clyde 1900-1950, Édimbourg, John Donald Publishers Ltd, 1992. 
les esprits et a fait entrer le combat pour UCS dans l'histoire des mouvements syndicaux.

\section{Émergence d'une nouvelle forme de lutte}

À la grève traditionnelle les ouvriers ont préféré la tactique du work-in, c'est-à-dire prendre le contrôle physique des lieux et s'emparer de l'outil de travail. Ainsi le 30 juillet 1971, les quatre divisions des chantiers navals d'UCS étaient sous le contrôle des ouvriers et des délégués. En quoi cette tactique fut-elle plus efficace qu'une grève? C'est ce qu'analysent Forster et Woolfson dans leur livre The Politics of the UCS Work-In. Ils suggèrent que le work-in eut un fort impact symbolique :

Voilà des hommes, pouvait-on dire, qui avaient été licenciés suite à une décision prise par le gouvernement, mais qui refusaient d'abandonner leur emploi. Ils continueraient de se présenter à leur poste de travail comme avant, montrant ainsi leur volonté et leur disponibilité à travailler même si officiellement ils n'étaient plus employés ${ }^{19}$. (Ibid., p. 171-172)

Avec une grève traditionnelle, les ouvriers auraient pu s'aliéner une partie de l'opinion publique et faire le jeu du gouvernement qui à la même époque, en août 1971, présentait son plan de réforme des syndicats, le très controversé Industrial Relations Act. En choisissant cette forme de résistance, les ouvriers ont envoyé un message fort au gouvernement et à la nation. Leur but n'était pas de paralyser l'économie mais de continuer le travail afin d'honorer le carnet de commandes et livrer les bateaux en temps et en heure. Non seulement ils montraient leur attachement à leur travail et leur rigueur, mais aussi leur dévouement à une entreprise qu'ils ne voulaient pas voir mourir. Contrairement aux revendications habituelles qui portaient sur les augmentations de salaire ou les conditions de travail, ces hommes demandaient juste le droit de poursuivre leur activité dans leur région. Ainsi «The Right to Work» devint le slogan emblématique de la lutte des ouvriers de la Clyde. À une époque où le chômage frôlait dangereusement le million, le caractère fédérateur de ce slogan a permis à beaucoup de s'identifier au sort des ouvriers d'UCS. Ce slogan a également permis de toucher les gens au-delà des frontières de la Clyde et au-delà du secteur des chantiers navals, d'où l'extraordinaire solidarité qui est née dans le monde ouvrier et syndical, mais aussi dans la classe

19. «Here were men, it could be argued, who had been made unemployed as a direct result of government action, but who refused to surrender their jobs. They would continue to turn up for work as previously, thereby demonstrating their willingness and availability for work even though officially they were no longer employed.» 
politique, Tony Benn et Harold Wilson étant plusieurs fois venus soutenir les ouvriers. Plus surprenant peut-être, les hommes d'église affichaient publiquement leur soutien aux ouvriers d'UCS et John Lennon et Yoko Ono avaient versé mille livres pour manifester leur soutien aux ouvriers (<http://www.gcal.ac.uk/radicalglasgow/chapters/ucs_workin.html>).

Une sympathie pour les ouvriers de la Clyde est née dans tout le pays qui a galvanisé les hommes et leur a permis de tenir le work-in pendant les seize mois de mobilisation. Tout le succès de leur mouvement dépendait de leur capacité à résister dans la durée. Il arrive toujours un moment d'essoufflement sur lequel les gouvernements comptent pour reprendre le dessus, mais les ouvriers d'UCS ont fait preuve d'une exceptionnelle cohésion et d'une exceptionnelle détermination. Preuve en est, le refus d'une grande majorité d'ouvriers de toucher leurs indemnités de licenciement. Buchan note qu'au début du mouvement beaucoup craignaient que «la tentation des indemnités de licenciement, accordées aux hommes qui partaient volontairement au chômage, ne se révèle plus forte que l'esprit de solidarité ${ }^{20} »$. Finalement, le sentiment de solidarité fut plus fort que l'appât du gain et sur les 8500 ouvriers menacés, on estime entre 350 et 400 les ouvriers qui ont perçu leurs indemnités et ont quitté l'entreprise (Buchan, p. 143). Jimmy Reid disait du communisme qu'il avait «cette capacité de développer chez les gens une forme d'incorruptibilité, une dévotion à la classe ouvrière et à la cause Socialiste, qui ne pouvait être intimidée ou achetée ${ }^{21}{ }^{\prime}$ (Reid, Reflections of a Clyde-Built Man, p. 47). Cette solidarité et cette fraternité parmi les ouvriers doivent en effet beaucoup à l'influence du communisme. Il faut savoir que le syndicalisme écossais fut fortement influencé à la fin du XIX ${ }^{\mathrm{e}}$ siècle par les théories socialistes et marxistes, et les discours des délégués syndicaux d'UCS, Jimmy Reid ou James Airlie, sont imprégnés de ces idéologies. Les communistes, de manière générale, étaient très influents dans le milieu des chantiers navals. Contrairement aux années 1920 et 1930 où leur présence était peu souhaitée voire même rejetée avec hostilité, dans les années 1960 et 1970 ils étaient accueillis avec enthousiasme car ils avaient la réputation d'être combatifs et dévoués à la cause des ouvriers (Knox, p. 290). Si le mouvement n'avait pas été mené par des délégués syndicaux communistes, la lutte pour UCS n'aurait peut-être pas connu le même succès.

20. «The great fear at the beginning was that the incentive of redundancy payments, being offered to men volunteering for redundancy would prove too strong an attraction to hold the solidarity.»

21. «this capacity to develop in people another incorruptibility, a dedication to the working class and the cause of Socialism, that cannot be intimidated nor bought». 
Cette unité au sein des ouvriers n'était pas gagnée d'avance, et ce en raison de la nature de l'organisation syndicale dans le milieu des chantiers navals. En effet, Forster et Woolfson soulignent son caractère fragmenté dû aux différents corps de métier qui co-existent dans les chantiers navals, chacun avec un apprentissage différent, des techniques différentes et des revendications différentes. Outre ce caractère fragmenté et corporatiste, c'est aussi un milieu particulièrement hiérarchisé entre les différents corps de métiers qui ne prédispose donc pas à l'unité et l'égalité (Forster et Woolfson, p. 132-133). Dans cet univers fragmenté et hiérarchisé, les délégués syndicaux communistes ont instauré une idée d'égalité parmi tous les ouvriers, quels que soient leurs qualifications ou leurs métiers et c'est là leur grande prouesse : créer une identité collective au-delà des différences de métiers et de hiérarchie. Par ailleurs, les communistes ont imposé un caractère autoritaire et intransigeant à leurs revendications. Ils étaient modérés dans leur comportement mais intransigeants dans leurs revendications : tout principe de négociation était rejeté car synonyme de concession et de capitulation. Le gouvernement n'était pas un partenaire, le gouvernement était un ennemi qu'il fallait vaincre. Lorsque Jimmy Reid refusa de serrer la main de John Davies, venu faire une visite sur le site d'UCS, ce n'était pas une provocation gratuite mais un acte politique fort comme le soulignent Forster et Woolfson :

Cela confirma la nature de la lutte. Les protestations sur l'économie écossaise ne pouvaient plus être ignorées avec des regrets polis. Le work-in des délégués syndicaux, ainsi que les conditions de leur résistance, avaient changé de manière décisive le rapport de force. L'opposition avait désormais pris une dimension directe, physique et inévitable qui, en même temps, donnait force et cohérence aux inquiétudes qui unissaient pratiquement toutes les couches de la société dans l'ouest de l'Écosse : la perte de contrôle sur l'économie, la destruction de l'industrie existante et l'érosion du droit le plus fondamental obtenu à l'après-guerre, le droit au travail ${ }^{22}$. (Ibid., p. 210)

Cette citation montre bien que la solidarité n'était pas fondée sur une idéologie commune, Reid et Airlie n’ont pas utilisé le communisme comme un élément fédérateur, ce n'est pas sur cette base-là qu'ils ont unifié le mouvement ouvrier. La solidarité était fondée sur une appartenance à

22. «It ratified the nature of the battle. Representations on the Scottish economy could no longer be dismissed with polite regret. The shop stewards' work-in, and the terms of their resistance, had decisively altered the balance of forces. Opposition had now assumed a direct, physical and unavoidable form which, at the same time, gave force and coherence to the concerns which united virtually all strata of society in the West of Scotland: the loss of control over the economy, the destruction of existing industry and the erosion of the most crucial political gain of the post-war period, the right to work. » 
la classe ouvrière et à une région, et il s'agit par conséquent davantage d'une solidarité identitaire qu'idéologique. Si les communistes étaient bien acceptés sur le terrain syndical, il n'en allait pas de même sur le terrain politique, il leur a donc été nécessaire d'effacer le discours communiste afin de s'assurer du soutien unanime de tous les ouvriers, des politiques, des hommes d'église et de la population. La réussite de ce mouvement doit beaucoup à un homme en particulier qui symbolise à lui seul le mouvement ouvrier pour la sauvegarde d'UCS : Jimmy Reid.

\section{Jimmy Reid : émergence d'un nouveau héros national}

Jimmy Reid entra au Parti communiste à l'âge de seize ans en 1948, gravit peu à peu les échelons et fut élu, en 1964, secrétaire du Parti communiste écossais. Lorsque la crise UCS éclata, Reid était le leader des délégués syndicaux parmi lesquels il commandait une grande influence, et il est indéniable que le succès de ce mouvement repose en grande partie sur son habileté à capturer l'état d'esprit, les angoisses et les aspirations de toute une communauté d'hommes et à inspirer un sentiment de loyauté et de fraternité parmi les ouvriers d'UCS. Jimmy Reid est devenu en quelques semaines le porte-parole des ouvriers licenciés, le héros de la classe ouvrière opprimée, la voix de l'Écosse sacrifiée. Dans un discours intitulé «Communism», Reid s'en prenait à la vision orthodoxe de l'histoire faite par les grands et les puissants qui «assigne la masse du peuple à un rôle de spectateur, à un rôle qui, en terme historique, les destitue de toute contribution au développement et au progrès de l'histoire de l'humanité ${ }^{3}$ » (Reid, p. 48). Avec la tactique du work-in, Reid voulait donner aux ouvriers un rôle actif, celui d'agents des mutations économiques et sociales, et voulait placer au cœur du processus de lutte des hommes dont la voix était trop souvent marginalisée et l'influence trop souvent niée. Le work-in était le symbole même de cette nouvelle forme d'action militante qui redéfinit les contours du pouvoir : en s'emparant et en contrôlant l'outil de production, les ouvriers changeaient de statut et devenaient à présent des interlocuteurs à écouter et respecter. Le work-in est l'application concrète du concept d'industrial democracy qui vit le jour à la fin du $\mathrm{XIX}^{\mathrm{e}}$ siècle et qui consiste à placer le contrôle et la gestion d'une entreprise dans les mains des ouvriers. Pendant seize mois, ce concept est devenu réalité et permettait aux ouvriers de sortir du schéma classique d'aliénation dont ils étaient victimes. Lors d'un discours prononcé à l'université de Glasgow en avril 1972, Reid définissait l'aliénation comme suit :

23. «consigns the great mass of the people to a spectator role, to a role where, in historical terms, they contribute nothing to any progressive development in mankind's history». 
C'est le cri d'hommes qui se sentent victimes de forces économiques arbitraires et incontrôlables. C'est la frustration du petit peuple qui se sent exclu du processus de décision. Le sentiment de détresse et de désespoir qui envahit les gens qui sentent, avec raison, qu'ils n'ont pas d'impact sur le cours de leur $\operatorname{destin}^{24}$. (Ibid., p. 99)

Deux niveaux de lecture émergent de cette citation. Reid évoque bien sûr le sort des ouvriers mais il évoque aussi le sort de l'Écosse aliénée et marginalisée vis-à-vis de l'Angleterre et du pouvoir de Londres. La classe ouvrière et l'Écosse partageaient le même sort et le même combat, ainsi lors de la manifestation du 18 août 1971, Reid réaffirmait conjointement la voix de la classe ouvrière et la voix de l'Écosse :

Les ouvriers britanniques se relèvent, se mettent debout et affirment leur dignité. Affirment de manière déterminée et disciplinée leur capacité à jouer un rôle dans les décisions qui sont prises dans ce pays [...]. Nous avons commencé par nous battre pour les emplois, et en l'espace de quelques jours nous nous battions pour l'Écosse, et pour la classe ouvrière britannique. Le véritable pouvoir de ce pays s'est forgé aujourd'hui à Clydeside, et sera forgé désormais dans les mines, dans les usines, dans les chantiers, dans les bureaux. Une fois que cette force est maîtrisée - disciplinée et déterminée - il n'existe aucune force au Royaume-Uni, ou même dans le monde, qui puisse l'arrêter ${ }^{25}$. (Ibid., p. 80-81)

Le discours ouvrier et le discours nationaliste sont indissociables chez Reid. Dans Reflections of a Clyde-Built Man, il décrit comment il entendit pour la première fois parler de l'histoire écossaise et de la nation écossaise au Parti communiste (ibid., p. 16). Les rapports entre la gauche et le nationalisme écossais sont bien connus et une des explications de ses liens intimes entre socialisme et nationalisme est, selon Reid, que la voix de l'Écosse ne peut être portée que par la classe ouvrière, car elle est l'âme même de l'Écosse :

24. «It is the cry of men who feel themselves the victims of blind economic forces beyond their control. It is the frustration of ordinary people excluded from the processes of decision-making. The feeling of despair and hopelessness that pervades people who feel with justification that they have no real say in shaping or determining their own destinies. »

25. «The workers of Britain are getting off their knees, getting on their feet and asserting their dignity. Asserting their abilities in a determined and disciplined way that they will have a say in the decision-making of this country [...]. We started off fighting for jobs, and in a matter of days we knew we were fighting for Scotland, and for the British working-class movement. The real power of this country has been forged today in Clydeside, and will be forged now in the pits, the factories, the yards and the offices. Once that force is given proper leadership - is disciplined and determinedthere is no force in Britain, or indeed the world, that can stand against it. » 
Aujourd'hui l'Écosse parle. Pas l'Écosse d'Edward Heath, Gordon Campbell, Sir Alec Douglas-Home — des lairds et de leurs larbins. Ils n'ont jamais représenté l'Écosse, la véritable Écosse, l'Écosse du peuple ouvrier ${ }^{26}$. (Ibid., p. 81)

Reid et ses camarades ont fait prendre conscience qu'il existait une voix ouvrière aussi bien qu'il existait une voix écossaise, ses voix trop souvent marginalisées ou méprisées se sont faites entendre et ont ainsi réveillé une prise de conscience en Écosse. Si les ouvriers étaient capables de prendre le contrôle de leur entreprise et d'agir sur leur destin, les Écossais, pareillement, étaient en capacité de prendre en main leur destin et de se gouverner de manière autonome sans la tutelle d'un gouvernement anglais condescendant et indifférent.

Reid a ainsi élargi les frontières du combat : bien plus qu'un mouvement syndical pour sauver des emplois, cette lutte est devenue un mouvement pour sauver l'Écosse et l'identité écossaise, car avec la disparition de l'industrie, c'est l'Écosse, ses traditions ouvrières et son identité qui disparaissaient peu à peu. Reid et ses collègues du Parti communiste ont sorti cette lutte ouvrière de la dialectique simpliste de la lutte des classes qui oppose les ouvriers au gouvernement, le capitalisme au communisme. Ils ont élargi le combat en un mouvement global qui allie défense de l'emploi, défense des droits des ouvriers, défense d'un savoir-faire et défense d'une identité. Mais il reste un dernier point sur lequel j'aimerais finir, car il me paraît important et peu d'ouvrages y font référence. J'aimerais considérer la dimension humaniste de ce combat pour la sauvegarde d'UCS, car il me semble que derrière la préservation de cette entreprise c'est aussi un combat pour préserver certaines valeurs qui s'est joué dans une société des années 1970 marquée par le matérialisme et le consumérisme.

\section{Résurgence d'un discours de valeurs : capitalisme versus humanisme}

La plupart des écrits sur cet épisode envisagent le mouvement ouvrier pour la sauvegarde d'UCS dans sa dimension syndicaliste ou nationaliste et il n'est pas fait référence à la dimension humaniste de cette lutte qui me semble pourtant primordiale. Les différents ouvrages évoquent le combat pour la défense de l'emploi, pour la défense de l'Écosse mais c'est aussi,

26. «Today Scotland speaks. Not the Scotland of Edward Heath, Gordon Campbell, Sir Alec Douglas-Home - of the Lairds and their lackeys. They have never represented Scotland, the real Scotland, the Scotland of the working people.» 
me semble-t-il, un combat pour préserver certaines valeurs dans une société où le profit, la rentabilité et la compétitivité semblaient être les maîtres mots au détriment des hommes et de leur bien-être. Lutter contre la fermeture d'UCS c'était aussi lutter contre un type de société, contre un esprit, et contre un système. L'humanisme était au cœur de ce combat, d'où sa portée universelle et la sympathie qu'il a inspiré dans tout le pays, toutes classes confondues, toutes régions confondues. Pour ma part, ce combat a pu «parler» à tous, car il a touché un aspect essentiel qui est la condition d'être humain et la manière dont on traite l'homme dans une société industrielle moderne. Le rapport Ridley ou les différents discours de John Davies niaient la part d'humanité dans l'industrie et n'envisageaient le monde industriel qu'à travers les chiffres, les bénéfices ou les pertes. Les ouvriers ont voulu réaffirmer que le monde industriel était avant tout un monde fait d'hommes, et que derrière la fermeture d'une entreprise, c'est le destin de milliers d'hommes qui se jouait. L'épisode UCS a vu la résurgence de valeurs fortes telles que la solidarité, la fraternité, l'égalité qui offraient une alternative au monde agressif et inhumain que proposait le Parti conservateur. Des hommes tels que Jimmy Reid ou James Airlie ont refusé de capituler devant les lois du libéralisme qui articulent le monde autour de la dialectique profit/perte. Le néo-libéralisme tend à déplacer le centre de gravité du débat politique vers l'économique au détriment de l'humain, or en luttant contre la politique du gouvernement Heath, ces hommes ont affirmé leur désir de résister à la déshumanisation progressive de la société et de replacer l'homme au centre du débat politique. Ces hommes étaient par conséquent convaincus de faire le bien et de se battre pour une cause juste et noble. Je partage l'avis de Buchan quand il écrit :

Mais la lutte représentait bien plus que la défense du droit au travail. En Écosse, elle avait une autre signification. Elle représentait l'ultime effort d'affirmer que le progrès de la technologie et de l'automatisation ne doivent pas être synonymes pour les hommes d'une perte de dignité dans le travail ${ }^{27}$. (Buchan, p. 146-147)

C'est pour cette raison aussi que la tactique du work-in a été préférée à la grève, car elle véhiculait une image positive des ouvriers au travail, tordant ainsi le cou à certains préjugés présentant les ouvriers en grève comme oisifs, perturbateurs et souvent fortement alcoolisés. La mission de Reid et de ses collègues syndicalistes était précisément de mettre fin à

27. «But the fight was more than just a defence of a right to be employed. In Scotland it involved something more. It was a last effort to assert that with the advance of technology and automation there must be no loss of dignity in men's employment. » 
ces préjugés sur la classe ouvrière, il en allait de la crédibilité de leur mouvement, d'où un discours ferme à l'égard des ouvriers et un protocole de bonne tenue à respecter :

Nous ne sommes pas des grévistes. Nous sommes des gens responsables et nous nous conduirons avec dignité et rigueur. Nous voulons travailler. Nous ne sommes pas des bêtes sauvages. Les vrais sauvages sont au 10 Downing Street et c'est ce gouvernement Tory. C'est la bande de voyous politiques la plus inhumaine qu'il m'ait été donné de rencontrer. À côté d'eux Al Capone et ses compères ressemblent à un groupe de boys scouts [...]. Il n’y aura pas d'hooliganisme, il n'y aura pas de vandalisme, il n'y aura pas de beuverie. Il en va de notre responsabilité que nous nous conduisions avec dignité et maturité $^{28}$. (Cité dans McGill, p. 102)

On perçoit dans les discours de Reid l'influence de l'église presbytérienne : rigueur, dignité, maturité, et surtout responsabilité, autant de termes utilisés à maintes reprises par Jimmy Reid et que l'on trouve également chez les hommes d'église qui, toutes confessions confondues, soutenaient les hommes d'UCS. Le 15 juin 1971, au lendemain de l'annonce de Davies de fermer UCS, ils avaient envoyé un télégramme à Heath lui demandant solennellement d'agir pour UCS, car une fermeture de l'usine «aurait des conséquences sociales et économiques telles qu'elles seraient inacceptables dans une société responsable ${ }^{29}$ » (cité dans Buchan, p. 62-63).

Des ouvriers licenciés au travail, des manifestations calmes et sans débordements, des hommes d'église qui soutiennent un mouvement syndicaliste mené par des communistes, l'épisode UCS a renversé les codes et les attentes. Les membres du gouvernement furent en effet sérieusement déstabilisés par l'attitude exemplaire des ouvriers. McGill explique que le Cabinet avait décidé de retarder la publication du rapport sur UCS commandé par Davies à la fin du mois de juillet, car le 14 juillet, date initialement prévue pour la sortie du rapport, les seules unités de l'armée disponibles étaient stationnées en Irlande du Nord. Ainsi Heath, Gordon Campbell — secrétaire d'État aux Affaires écossaises — et Davies préférèrent attendre que les troupes soient revenues en Grande-Bretagne

28. «We are not strikers. We are responsible people and we will conduct ourselves with dignity and discipline. We want to work. We are not wild cats. The real wild cats are in 10 Downing Street as represented by this Tory Government. They are the hardest-faced bunch of political gangsters I have ever met. They make $\mathrm{Al}$ Capone and his gunmen look like a troop of Boy Scouts [...]. There will be no hooliganism, there will be no vandalism, there will be no bevying. It is our responsibility to conduct ourselves with dignity and maturity.»

29. «would have such social and economic consequences as to be totally unacceptable in a responsible society». 
avant de publier officiellement le rapport (McGill, p. 101). Finalement, le gouvernement fut pris au dépourvu, car rarement mouvement ouvrier aura été aussi pacifique. Si violence il y eut, c'est uniquement dans les mots très durs contre le gouvernement, mais jamais dans les actes. Reid le savait bien : la violence rend le message inaudible et fait le jeu du gouvernement. Or, tout le génie de Reid est d'avoir réussi à renverser les rôles, car dans ce conflit c'est le gouvernement qui apparaissait comme révolutionnaire, dogmatique et inhumain. Heath, Ridley et Davies étaient dépeints comme des monstres froids, sans cœur et sans âme, prêts à laisser mourir une région au nom d'une politique capitaliste. Bien sûr les accusations étaient parfois un peu caricaturales et abusives, l'émotion y étant pour beaucoup, mais la violence des propos contre le gouvernement ne faisait que répondre à la violence des propos d'hommes comme Ridley ou Davies.

Ridley et Davies étaient des hommes d'affaires, des gestionnaires qui regardaient cette affaire uniquement sous l'angle financier, mais on ne peut pas accuser Heath de la même indifférence. Heath n'est pas resté insensible face au sort de ces hommes. Heath était un homme politique guidé par une éthique personnelle fortement ancrée dans des valeurs chrétiennes et une éthique politique inspirée de la philosophie du torysme One Nation. Les valeurs portées par ces hommes l'ont indéniablement touché et ont certainement contribué à le faire changer d'avis, après tout n'était-il pas l'un des fondateurs du groupe One Nation qui avait rédigé en 1959 The Responsible Society ${ }^{30}$. Comme Harold Macmillan avant lui, Heath était issu de la tradition Tory d'après-guerre qui honnissait le chômage et la pauvreté. Heath n'était pas dans une optique de confrontation; il n'était pas l'ennemi des syndicats et malgré un discours qui pouvait paraître agressif et radical, il était le plus réceptif et le plus sincèrement préoccupé par le sort des hommes d'UCS. Jimmy Reid ne s'était pas trompé à ce sujet, lui qui déclarait avoir «une certaine tendresse pour le Premier ministre, notamment au regard de la politique menée par son successeur, Margaret Thatcher ${ }^{31} »$.

En effet on peut penser que l'issue du conflit aurait été sensiblement différente si les hommes d'UCS avaient eu en face d'eux Margaret Thatcher. Heath a mis du temps avant de changer d'avis, car dans cette affaire il s'est retrouvé coincé dans une logique politique — il avait été élu sur un programme de désengagement de l'État — et une logique

30. Voir The Conservative Party Archives, PUB 167/21, One Nation Group, The Responsible Society, Londres, Conservative Political Centre, 1959.

31. «a soft spot for the Tory Prime Minister, particularly in the light of the policies of his successor, Margaret Thatcher» (<http://news.bbc.co.uk/1/hi/programmes/uk_confidential/1717110.stm>). 
politicienne — ne pas perdre la face vis-à-vis de son parti — , d'où son long silence qui a été interprété à tort comme de l'indifférence. Heath s'est retrouvé confronté à un dilemme entre considérer la question uniquement sous l'angle économique — fermer UCS — ou sous l'angle humain - sauver UCS et injecter des fonds publics. Finalement, sa vraie nature a resurgi et son attachement au torysme One Nation a pris le dessus et Heath a fait marche arrière. Le 28 février 1972, Davies annonçait à la Chambre des communes une aide massive à UCS à hauteur de 35 millions de livres (House of Commons Debate, Hansard, 2 août 1971, vol. 832 / col. 52). Avec l'aide à UCS, c'est toute une politique qui s'effondrait. Le gouvernement conservateur avait voulu faire d'UCS le symbole d'une politique de fermeté à l'égard des «canards boiteux», il sera finalement le symbole des retours en arrière initiés par le gouvernement à partir de 1972.

\section{Conclusion}

En 1976, Keith Joseph écrivait en préface d'un rapport sur Upper Clyde Shipbuilders que la gestion de la crise par Heath

[...] avait permis à un apparatchik communiste notoire, Jimmy Reid, de devenir du jour au lendemain un héros national, elle coûta des dizaines de millions de livres au contribuable britannique, et c'était loin d'être fini, et ne résolut en rien les problèmes liés à la construction navale dans la région ${ }^{32}$ (Broadway, Upper Clyde Shipbuilders, p. Ix).

Peut-être Joseph n'avait-il pas tout à fait tort, et bien que les propos soient durs ils soulignent avec justesse que la victoire des ouvriers d'UCS ne fut qu'une pause, un court instant de répit dans un processus inexorable de déclin de l'industrie écossaise. Il existe des changements sociétaux contre lesquels il est impossible de lutter et la victoire d'UCS ne faisait que remettre à plus tard une interrogation nécessaire sur les problèmes structurels de l'économie écossaise. Mais n'existe-t-il pas une autre forme de victoire derrière cet épisode UCS? La victoire du mouvement ouvrier représente de manière symbolique la victoire de l'Écosse sur l'Angleterre. Par une heureuse coïncidence de l'histoire, les Écossais avaient une fois de plus vaincu Édouard, renouant ainsi avec un des épisodes les plus glorieux de leur histoire. Et finalement n'est-ce pas là la véritable victoire

32. «[...] had enabled a committed Communist apparatchik, Jimmy Reid, to become a national figure overnight, it cost the British taxpayer tens of millions of pounds, with more to come, and left all the problems of shipbuilding in the region unsolved.» 
d'UCS, d'avoir fait resurgir une fierté écossaise malmenée par les difficultés économiques, d'avoir fait resurgir une conscience écossaise étouffée par la centralisation du pouvoir à Londres, d'avoir aidé en somme à la résurgence d'un nationalisme écossais? Car en se battant pour leur usine, les ouvriers se battaient aussi pour leur nation, le sort de l'un étant intimement lié au sort de l'autre. UCS est alors devenu un symbole, un symbole de résistance et de lutte, et plus largement le symbole d'une victoire contre la marginalisation, réhabilitant ainsi la place centrale de l'Écosse et des Écossais dans le débat national.

\section{Références bibliographiques}

\section{Sources primaires}

Archives

Archives du Parti conservateur

PUB 167/21, One Nation Group, The Responsible Society, Londres, Conservative Political Centre, 1959.

PUB 106/4, Frank Broadway, Upper Clyde Shipbuilders: A Study of Government Intervention in the Industry... and the Way the Money Goes, Londres, Centre of Political Studies, 1976.

Archives nationales

PREM 15 : Prime Minister's paper and correspondence.

Débats parlementaires

House of Commons Debate

Hansard, 4 novembre 1970, volume 805.

-, 14 juin 1971, volume 819 .

—, 2 août 1971, volume 832.

$\underline{\text { Presse }}$

The Glasgow Herald, 12 juin 1971.

The Guardian, 6 mai 1970.

\section{Sources secondaires}

Buchan Alasdair, The Right to Work: The Story of the Upper Clyde Confrontation, Londres, Calder \& Boyars, 1972.

Civardi Christian, Le Mouvement ouvrier écossais, 1900-1931. Travail, culture, politique, Strasbourg, Presses universitaires de Strasbourg, 1997, 464 p. 
Devine Tom M., The Scottish Nation 1700-2000, Londres, Penguin Books, 1999.

Duncan Robert et MaIvor Arthur (eds), Militant Workers: Labour and Class Conflict on the Clyde 1900-1950, Édimbourg, John Donald Publishers Ltd, 1992.

Forster John et WoOLFson Charles, The Politics of the UCS Work-In: Class Alliances and the Right to Work, Londres, Lawrence \& Wishart, 1986.

Knox William W., Industrial Nation: Work, Culture and Society in Scotland, 1800-Present, Édimbourg, Edinburgh University Press, 1999.

MaGill Jack, Crisis on the Clyde, Londres, Davis Poynter, 1973.

McLean Iain, The Legend of Red Clydeside, Édimbourg, John Donald Publishers Ltd, 1983.

ReID Jimmy, Reflections of a Clyde-Built Man, Londres, Souvenir Press (E\&A) Ltd, 1976.

\section{Sites Internet}

<http://news.bbc.co.uk/1/programmes/uk_confidential/1717110.stm> [consulté le 17 mars 2008].

<http://www.gcal.ac.uk/radicalglasgow/chapters/ucs_workin.html> [consulté le 23 mars 2008]. 